\title{
Effects of Force Fields on the Equilibrium State of 5,7-Dihydroxy-4-MethylCoumarin Comparative Study of Molecular and Langevin Dynamic Simulations
}

\author{
Radia Mahboub ${ }^{1} \&$ Mourad Mahboub ${ }^{1}$ \\ ${ }^{1}$ Faculty of Sciences, Abou Bakr Belkaid University, B.P. 119, Tlemcen, Algeria \\ Correspondence: Radia Mahboub, Department of Chemistry, Faculty of Sciences, Abou Bakr Belkaid University, \\ B.P. 119, Tlemcen 13000, Algeria. E-mail: radiamahboub@yahoo.com
}

Received: March 26, 2012

doi:10.5539/mas.v6n5p 100
Accepted: April 23, $2012 \quad$ Online Published: May 1, 2012

URL: http://dx.doi.org/10.5539/mas.v6n5p100

\begin{abstract}
We have studied the influence of the two force fields Amber and MM+ in both gas and solution environments. Molecular Dynamic (MD) and Langevin Dynamic (LD) simulations of the 5,7-dihydroxy-4-methylcoumarin were performed with the an efficient program. The geometries, interaction energies, bonds, angles, stretch-bends, electrostatic and the Van der Waals (VDW) interactions were carried out in solution and gas phases. The results show that MD and LD simulations are identical. This comparative study shows that the coumarin acquires the low-energy and has the dipolar characteristic under the MM+ field by using the both methods. This molecule reaches its high stable conformation state in solution environment. So, under $\mathrm{MM}+$ field, the simulation gives best results.
\end{abstract}

Keywords: AMBER, MM+, molecular dynamics (MD), langevin dynamics (LD), coumarin

\section{Introduction}

Coumarins are an important class of heterocyclic organic compounds (Borges et al., 2005). They have attracted great interest due to their importance in synthetic organic chemistry, and to their possession of diverse pharmacological properties (Hoult \& Payá, 1996). Coumarins consist of a carbon-carbon double bond which is fixed as trans conformation as in trans-stilbene through a lactone structure. From the molecular structure result a strong fluorescence and photostability in most of coumarin derivatives.

In the late of 1950, Wheelock has shown that substitutions on the coumarin structure shifted the fluorescence band. For example, adding a methyl group to the 4-position of 7-hydroxy- or 7-methoxycoumarin moves the fluorescence spectra. Addition of electron-repelling groups in the 4-, 6-, or 7-position or electron-attracting groups in the 3-position shifts the fluorescence band to longer wavelengths.

As consequence, 4-methylcoumarins have been found to possess choleretic, analgesic, anti-spermatogenic, anti-tubercular and diuretic properties (Goel et al., 2007; Pedersen et al., 2007). Hydroxyl derivatives of 4-methylcoumarin are used as optical brightening, dispersed fluorescent lasers (Shan \& Xiao, 2004; Wheelock, 1959). Recently, they are employed as potential bio-antioxidants agents (Borges et al., 2005; Hoult \& Payá, 1996; Kumar et al., 2005; Kancheva, 2009).

In our earliest work, we have studied the different properties of some coumarin systems by Molecular dynamics (Mesli \& Mahboub, 2010). From these results, we are interested in 5,7-dihydroxy-4-methylcoumarin. For this system, we apply Molecular dynamics (MD) and Langevin dynamics (LD) methods. In this article, we detail the method of simulation using AMBER and MM+ as force fields. We will present here recent results both in water and gas environments and we give geometry optimization, energies and dihedrals angles properties.

In the present work, we describe and characterize the molecular structure of 5,7-dihydroxy-4-methylcoumarin by two methods: MD and LD simulations using two force fields: AMBER and MM+. We discuss the computational chemistry results of the coumarin compound then we compare the two calculations methods and force fields effects.

In section 2, we describe the materials and methods used in this study. In the next section, we have detailed the molecular calculations: i) geometry optimization, and ii) dynamic simulations. In the second part, we discuss the evolution of: i) energies, ii) dihedrals angles, iii) temperatures, and iv) geometry optimization in both vacuum 
and water environments. We present the calculation results obtained with the two force fields: AMBER and $\mathrm{MM}+$ by Molecular and Langevin dynamics, then we compare all this results. In the last section we give the conclusion.

\section{Materials and Methods}

Molecular dynamics (MD) is a useful technique to study the kinetics and thermodynamics properties of proteins and other biomolecules. The success of MD is the study process energy of organic compounds as protein, heterocyclic, and important conformational changes in a small time step size.

Solvent effects can be modeled by Langevin dynamics then a molecular system in a liquid environment can be studied. These simulations can be much faster than molecular dynamics. Langevin dynamics simulates the effect of molecular collisions and the resulting dissipation of energy that occurs in real solvents, without explicitly including solvent molecules. This is accomplished by adding a random force and a frictional force to each atom at each time step (van Gunsteren \& Berendsen, 1990; Karplus \& Petsko, 1990). Because of the implicit treatment of the solvent, this method is particularly well-suited for studying large molecules in solution (Sabbaghzadeh et al., 2010).

Molecular mechanics (MM) force field is a combination of Coulomb and Lennard-Jones interactions to describe all non-bonded interactions (Ponder \& Case, 2003). The potential energy is simple and depends on a large number of empirical parameters, which must be obtained from $a b$ initio calculations of the electronic structure in small molecules and experimental data. Though AMBER utilizes implicit hydrogen bonding terms (MacKerrell et al., 1998; Cornell et al., 1995; Weiner et al., 1984).

In vacuum, the system was simulated using Molecular and Langevin dynamics (MD and LD) with 0.0001 ps step. For the LD simulation, the frictional coefficient was chosen to $0.05 \mathrm{ps}^{-1}$. Temperature was kept constant at $300 \mathrm{~K}$. The starting temperature was taken at $100 \mathrm{~K}$ and the step at $20 \mathrm{~K}$.

In water, simulations, the system was placed in a box $(20 \times 20 \times 20 \AA)$ containing one molecule of 5,7-dihydroxy-4-methylcoumarin and 254 water molecules and cut-off $4 \AA$. Optimization of the molecule was realized in periodic boundary conditions (PBC). The compound was solvated by added water molecules. The systems were first energy minimized steps with the conjugate gradient algorithm. Then, the position-restrained MD and LD simulation were run 0.5 ps.

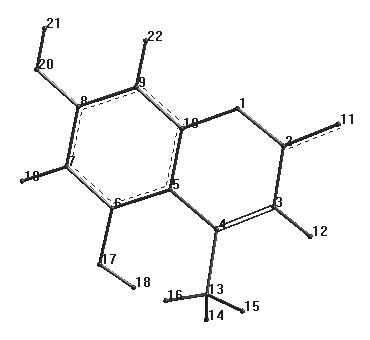

(a)

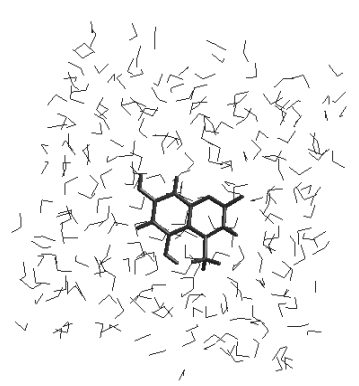

(b)

Figure 1. Geometry structure of 5,7-dihydroxy-4-methylcoumarin: (a)In vacuum, (b)In water (254 TIP3P water molecules)

\section{Results and Discussion}

\subsection{Geometry Optimization}

The geometry optimizations of 5,7-dihydroxy-4-methylcoumarin are realized in vacuum and in water (in PBC with a 253 TIP3P water molecules); using two force fields: AMBER and MM+. The results are given in Table 1. As consequence, the solvated system has lower energies than the isolated structure with both the force fields. 
Table 1. Geometry optimization properties of 5,7-dihydroxy-4-methylcoumarin at $300 \mathrm{~K}$ with $\mathrm{MM}+$ and AMBER before dynamic simulations

\begin{tabular}{|c|c|c|c|c|c|c|c|c|c|c|}
\hline \multirow{3}{*}{ State } & \multicolumn{10}{|c|}{ Geometry } \\
\hline & Force & Bond & Angle & Dihedral & VDW & Stretch-bend & H-bond & Electrostatic & Energy & Gradient \\
\hline & field & $(\mathrm{Kcal} / \mathrm{mol})$ & $(\mathrm{Kcal} / \mathrm{mol})$ & $(\mathrm{Kcal} / \mathrm{mol})$ & $(\mathrm{Kcal} / \mathrm{mol})$ & (Kcal/mol) & $(\mathrm{Kcal} / \mathrm{mol})$ & $(\mathrm{Kcal} / \mathrm{mol})$ & $(\mathrm{Kcal} / \mathrm{mol})$ & (Kcal/Å.mol) \\
\hline \multirow[t]{2}{*}{ Isolated } & AMBER & 0.5981 & 1.341 & 1.9394 & 5.5091 & - & -0.0001 & 0 & 9.3881 & 0.0919 \\
\hline & $\mathrm{MM}+$ & 1.0137 & 4.2602 & -8.4056 & 8.16342 & -0.0507 & - & 0.3398 & 5.3208 & 0.0996 \\
\hline \multirow{2}{*}{ Solvated } & AMBER & 33.8598 & 37.1948 & 2.5932 & 134.435 & - & -0.0002 & -4143.67 & -3935.5886 & 0.0969 \\
\hline & $\mathrm{MM}+$ & 1.0684 & 9.4177 & -8.2320 & -203.361 & -0.1741 & - & -481.124 & -682.4055 & 0.0832 \\
\hline
\end{tabular}

Trans (gauche) conformation of the coumarin structure is confirmed by the two dihedral angles $\left(\mathrm{C}_{10}-\mathrm{O}_{1}-\mathrm{C}_{2}-\mathrm{O}_{11}\right.$ (coco), and $\left.\mathrm{C}_{4}-\mathrm{C}_{3}-\mathrm{C}_{2}-\mathrm{O}_{11}\right)$ and the distance bonds. In the solvated coumarin, the torsion angle $\mathrm{C}_{4}-\mathrm{C}_{3}-\mathrm{C}_{2}-\mathrm{O}_{11}$ changes. So, we obtained an anhedral angle value of $-176.406^{\circ}$ (Table 2a). This situation is due of the strong collisions between coumarin and water under AMBER.

Table 2a. Structural properties of 5,7-dihydroxy-4-methylcoumarin with AMBER

\begin{tabular}{|c|c|c|c|c|c|c|c|c|c|c|c|}
\hline \multicolumn{12}{|c|}{ Coumarin } \\
\hline \multicolumn{6}{|c|}{ Isolated } & \multicolumn{6}{|c|}{ Solvated } \\
\hline Angle & $\Theta\left({ }^{\circ}\right)$ & Dihedral & $\Phi\left(^{\circ}\right)$ & Bond & $\mathrm{D}(\AA)$ & Angle & $\Theta\left({ }^{\circ}\right)$ & Dihedral & $\Phi\left(^{\circ}\right)$ & Bond & $\mathrm{D}(\AA)$ \\
\hline $10-1-2$ & 121.855 & & & $14-19$ & 2.635 & $10-1-2$ & 122.067 & & & $14-19$ & 2.643 \\
\hline $1-2-3$ & 119.921 & & & $13-18$ & 2.588 & $1-2-3$ & 119.652 & & & $13-18$ & 2.604 \\
\hline $11-2-3$ & 120.323 & & & $11-22$ & 2.559 & $11-2-3$ & 120.815 & & & $11-22$ & 2.576 \\
\hline $1-2-11$ & 119.746 & & & $13-15$ & 2.520 & $1-2-11$ & 119.510 & & & $13-15$ & 2.617 \\
\hline $22-3-2$ & 119.529 & & & $13-16$ & 3.814 & $22-3-2$ & 120.010 & & & $13-16$ & 3.843 \\
\hline $6-13-20$ & 113.535 & $10-1-2-11$ & 179.706 & $13-17$ & 2.507 & $6-13-20$ & 113.235 & $10-1-2-11$ & 172.303 & $13-17$ & 2.457 \\
\hline \multirow[t]{3}{*}{$8-14-21$} & 113.372 & $4-3-2-11$ & 179.735 & $14-18$ & 2.637 & $8-14-21$ & 113.320 & $4-3-2-11$ & -176.406 & $14-18$ & 2.630 \\
\hline & & & & 1011 & 3.48 & & & & & 1011 & 3.473 \\
\hline & & & & 411 & 3.556 & & & & & 411 & 3.548 \\
\hline
\end{tabular}

Table 2b. Structural properties of 5,7-dihydroxy-4-methylcoumarin with MM+

\begin{tabular}{|c|c|c|c|c|c|c|c|c|c|c|c|}
\hline \multicolumn{12}{|c|}{ Coumarin } \\
\hline \multicolumn{6}{|c|}{ Isolated } & \multicolumn{6}{|c|}{ Solvated } \\
\hline Angle & $\Theta\left({ }^{\circ}\right)$ & Dihedral & $\Phi\left(^{\circ}\right)$ & Bond & $\mathrm{D}(\AA)$ & Angle & $\theta\left(^{\circ}\right)$ & Dihedral & $\Phi\left(^{\circ}\right)$ & Bond & $\mathrm{D}(\AA)$ \\
\hline $10-1-2$ & 119.341 & & & $14-19$ & 2.640 & $10-1-2$ & 119.291 & & & $14-19$ & 2.644 \\
\hline $1-2-3$ & 120.407 & & & $13-18$ & 2.572 & $1-2-3$ & 120.676 & & & $13-18$ & 2.573 \\
\hline $11-2-3$ & 120.330 & & & $11-22$ & 2.491 & $11-2-3$ & 120.013 & & & $11-22$ & 2.486 \\
\hline $1-2-11$ & 118.966 & & & $13-15$ & 2.472 & $1-2-11$ & 119.307 & & & $13-15$ & 2.449 \\
\hline $22-3-2$ & 118.363 & & & $13-16$ & 3.762 & $22-3-2$ & 120.013 & & & $13-16$ & 3.753 \\
\hline $6-13-20$ & 120.236 & $10-1-2-11$ & 179.927 & $13-17$ & 2.447 & $6-13-20$ & 120.253 & $10-1-2-11$ & 179.890 & $13-17$ & 2.447 \\
\hline \multirow[t]{3}{*}{$8-14-21$} & 120.147 & $4-3-2-11$ & 179.986 & $14-18$ & 2.637 & $8-14-21$ & 119.943 & $4-3-2-11$ & 179.681 & $14-18$ & 2.631 \\
\hline & & & & 1011 & 3.425 & & & & & 1011 & 3.427 \\
\hline & & & & 411 & 3.451 & & & & & 411 & 3.448 \\
\hline
\end{tabular}


In vacuum, the minimum potential energies $E_{M D \text {,min }}$ and $E_{L D \text {,min }}$ calculated for the geometry optimization using AMBER and MM+ force fields are identical (respectively $9.30 \mathrm{Kcal} / \mathrm{mol}$, and $5.31 \mathrm{Kcal} / \mathrm{mol}$ ). We note that the dihedral factor in potential energy is big in MD simulation than in LD when using AMBER field (table 3a). For the last situation, the system considers the friction coefficient which affects the structure geometry of molecule.

In water, we have obtained the same minimum potential energies $\mathrm{E}_{\mathrm{MD} \text {,min }}$ and $\mathrm{E}_{\mathrm{LD} \text {,min }}$ for both $\mathrm{AMBER}$ and $\mathrm{MM}+$ force fields. Variations are visible for bonds, angle, and dihedral energies. The much important difference is happened in the Van der Waals energies.

In MD or LD calculations, the collisions between solute-solvent and solvent-solvent are very important and increase the energy in MM+ field than in AMBER one where the rubbing are present due to the friction coefficient $(\mathrm{VDW}=5.4, \mathrm{VDW}=8.16 \mathrm{Kcal} / \mathrm{mol})($ Tables $3 \mathrm{a}, \mathrm{b})$.

Table 3a. Geometry optimization properties of 5,7-dihydroxy-4-methylcoumarin at $300 \mathrm{~K}$ with $\mathrm{MM}+$ and AMBER after LD and MD simulations in vacuum

\begin{tabular}{|c|c|c|c|c|c|c|c|c|c|c|}
\hline Method & $\begin{array}{l}\text { Force } \\
\text { field }\end{array}$ & $\begin{array}{c}\text { Bond } \\
(\mathrm{Kcal} / \mathrm{mol})\end{array}$ & $\begin{array}{c}\text { Angle } \\
(\mathrm{Kcal} / \mathrm{mol})\end{array}$ & $\begin{array}{c}\text { Dihedral } \\
\text { (Kcal/mol) }\end{array}$ & $\begin{array}{c}\text { VDW } \\
(\mathrm{Kcal} / \mathrm{mol})\end{array}$ & $\begin{array}{l}\text { Stretch-bend } \\
(\mathrm{Kcal} / \mathrm{mol})\end{array}$ & $\begin{array}{c}\text { H-bond } \\
\text { (Kcal/mol) }\end{array}$ & $\begin{array}{l}\text { Electrostatic } \\
(\mathrm{Kcal} / \mathrm{mol})\end{array}$ & $\begin{array}{c}\text { Energy } \\
\text { (Kcal/mol) }\end{array}$ & $\begin{array}{c}\text { Gradient } \\
\text { (Kcal/ Å.mol) }\end{array}$ \\
\hline \multirow{2}{*}{ MD } & AMBER & 0.5646 & 1.2342 & 2.1058 & 5.4013 & - & -0.0001 & 0 & 9.3058 & 0.0887 \\
\hline & $\mathrm{MM}+$ & 1.0135 & 4.2698 & -8.4277 & 8.1692 & -0.0492 & - & 0.3404 & 5.3160 & 0.0935 \\
\hline \multirow{2}{*}{ LD } & AMBER & 0.5827 & 1.3156 & 1.9592 & 5.4433 & - & -0.0001 & 0 & 9.3007 & 0.0891 \\
\hline & MM+ & 1.0068 & 4.2728 & -8.4283 & 8.1713 & -0.0481 & - & 0.3402 & 5.3149 & 0.0907 \\
\hline
\end{tabular}

Table 3b. Geometry optimization properties of 5,7-dihydroxy-4-methylcoumarin at $300 \mathrm{~K}$ with $\mathrm{MM}+$ and AMBER after LD and MD simulations in water (254 TIP3P water molecules)

\begin{tabular}{|c|c|c|c|c|c|c|c|c|c|c|}
\hline Method & Force field & $\begin{array}{c}\text { Bond } \\
(\mathrm{Kcal} / \mathrm{mol})\end{array}$ & $\begin{array}{c}\text { Angle } \\
(\mathrm{Kcal} / \mathrm{mol})\end{array}$ & $\begin{array}{c}\text { Dihedral } \\
\text { (Kcal/mol) }\end{array}$ & $\begin{array}{c}\text { VDW } \\
(\mathrm{Kcal} / \mathrm{mol})\end{array}$ & $\begin{array}{l}\text { Stretch-bend } \\
(\mathrm{Kcal} / \mathrm{mol})\end{array}$ & $\begin{array}{c}\text { H-bond } \\
\text { (Kcal/mol) }\end{array}$ & $\begin{array}{l}\text { Electrostatic } \\
(\mathrm{Kcal} / \mathrm{mol})\end{array}$ & $\begin{array}{c}\text { Energy } \\
\text { (Kcal/mol) }\end{array}$ & $\begin{array}{c}\text { Gradient } \\
\text { (Kcal/ Å.mol }\end{array}$ \\
\hline \multirow{2}{*}{ MD } & AMBER & 0.5762 & 1.2860 & 2.0122 & 5.4313 & - & -0.0001 & 0 & 9.3056 & 0.0964 \\
\hline & $\mathrm{MM}+$ & 1.0118 & 4.2896 & -8.4264 & 8.1508 & -0.0484 & - & 0.3396 & 5.3170 & 0.0911 \\
\hline \multirow{2}{*}{ LD } & AMBER & 0.5815 & 1.3126 & 2.0039 & 5.4086 & - & -0.0001 & 0 & 9.3066 & 0.0960 \\
\hline & $\mathrm{MM}+$ & 1.0197 & 4.2836 & -8.4268 & 8.1488 & -0.0480 & - & 0.3397 & 5.3170 & 0.0914 \\
\hline
\end{tabular}

\subsection{Dynamic Simulation}

Dynamic simulations were accomplished at constant temperature, using two force fields: AMBER and MM+. The conjugate gradient (Polak-Ribiere) algorithm was preferred because for the constant dielectric. We have calculated the coumarin dynamic properties in both gas and water. We have employed the TIP3P water molecules model, and chosen the bath relaxation time equals to $0.1 \mathrm{ps}$. The simulation temperature was fixed to $300 \mathrm{~K}$ and the step at $20 \mathrm{~K}$. The run time was $0.5 \mathrm{ps}$, the step size was $0.0001 \mathrm{ps}$ and the heat time was $0.1 \mathrm{ps}$. Here, we study the evolution of: i) energies, ii) dihedrals angles, iii) temperatures, and iv) energy deviations in both vacuum and water environments.

At first, we have calculated all energies for the two force fields of AMBER and MM+ simulation then presented them in Figures 2, 3, 4, and 5. The average energies are in good agreement with the simulation accuracy. The sampling results of step-size of MD and LD methods are presented in Figures (a) and (b), respectively.

With AMBER, the total energies (ETOT) are the same in MD and LD simulations in vacuum environment. After equilibration, the MD simulation becomes more stable (respectively $45.25 ; 45.06 \mathrm{Kcal} / \mathrm{mol}$ ). The potential energy (EPOT) and the kinetic energy (EKIN) illustrate fluctuations in both methods and vary around (24.77, 20.47) $\mathrm{Kcal} / \mathrm{mol}$ for MD, and $(23.19,21.86) \mathrm{Kcal} / \mathrm{mol}$ for LD (Figure 2). In LD simulation, we observe that the trajectory of potential energy has attained a minimum around $0.3 \mathrm{ps}(18.80 \mathrm{Kcal} / \mathrm{mol})$. In the same time, the kinetic energy provides an opposite situation i.e. its reach a maximum $(27.10 \mathrm{Kcal} / \mathrm{mol})$. 


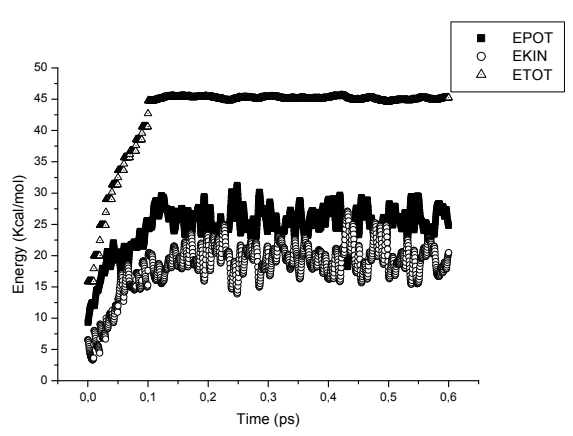

$\left(a_{1}\right)$

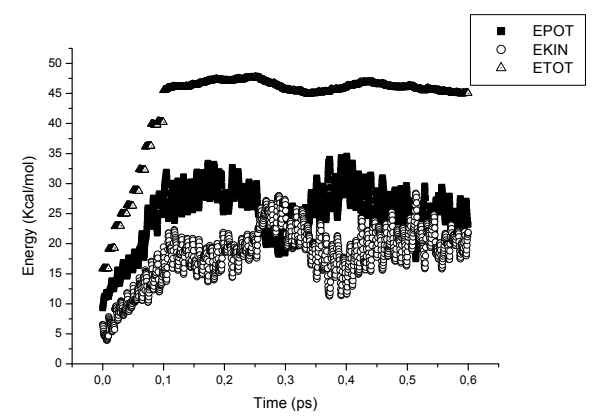

$\left(b_{1}\right)$

Figure 2. Evolution of energies for 5,7-dihydroxy-4-methylcoumarin in vacuum with AMBER: (a)MD, (b)LD

With $\mathrm{MM}+$ (in gas), total energy in MD simulation is little high than in LD and much stable after equilibrium time (MD: 42.29, LD: $40.34 \mathrm{Kcal} / \mathrm{mol}$ ). The kinetic energies have the same value in the two simulations (MD: $16.16,16.51 \mathrm{Kcal} / \mathrm{mol}$ ). However, potential energies is high for MD than for LD (MD: 26.12, LD: 23.82 $\mathrm{Kcal} / \mathrm{mol}$ ) (Figure 3).

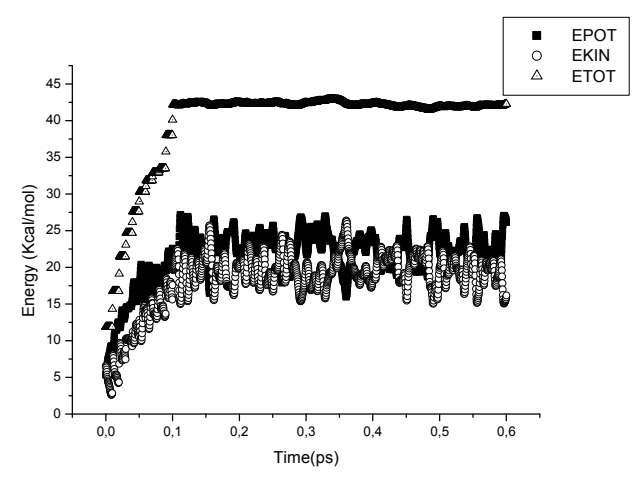

$\left(a_{1}\right)$

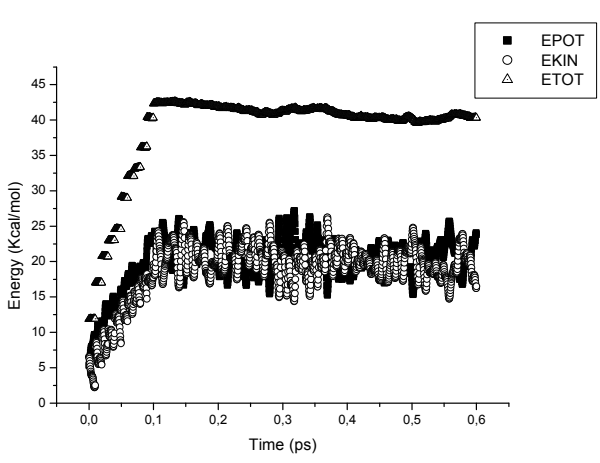

$\left(b_{1}\right)$

Figure 3. Evolution of energies for 5,7-dihydroxy-4-methylcoumarin in vacuum with MM+: (a)MD, (b)LD

Under the force field AMBER in water, the evolution of total energy in MD simulation is much stable and little big than in LD one. In MD EPOT energy is greater than in LD. The situation is reversed for kinetic energies (MD: ETOT 41.70, EPOT 23.08, EKIN $18.61 \mathrm{Kcal} / \mathrm{mol}$; LD: ETOT 41.38, EPOT 22.90, EKIN $18.48 \mathrm{Kcal} / \mathrm{mol}$ ) (Figure 4).

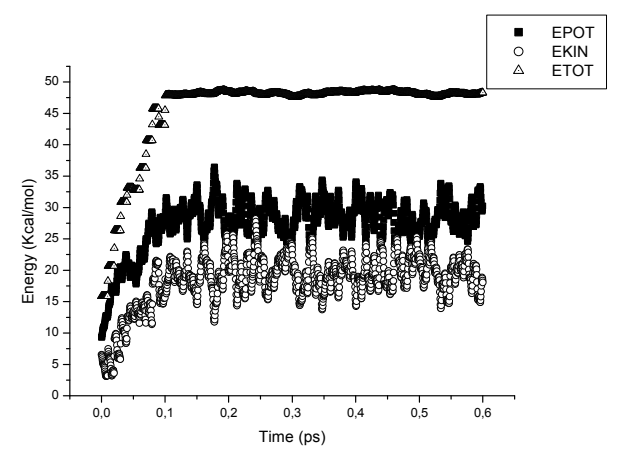

$\left(a_{1}\right)$

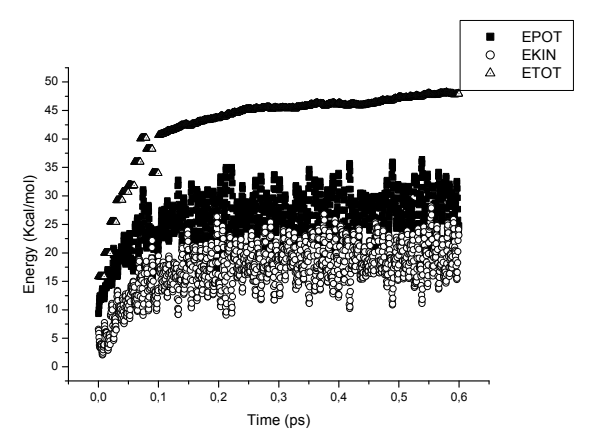

$\left(b_{1}\right)$

Figure 4. Evolution of energy for 5,7-dihydroxy-4-methylcoumarin in TIP3P water molecules with AMBER:

(a)MD, (b)LD 
Under the force field MM+ in solution, the position of all energies stay unaltered (MD: ETOT 40.12, EKIN 22.62, EPOT $17.50 \mathrm{Kcal} / \mathrm{mol}$; LD: ETOT 39.77, EKIN 21.88, EPOT 17.89, Kcal/mol). All energies are very stables at time greater than $0.1 \mathrm{ps}$ for both simulations. Here, the fluctuations disappear in spite of interactions between molecules (Figure 5).

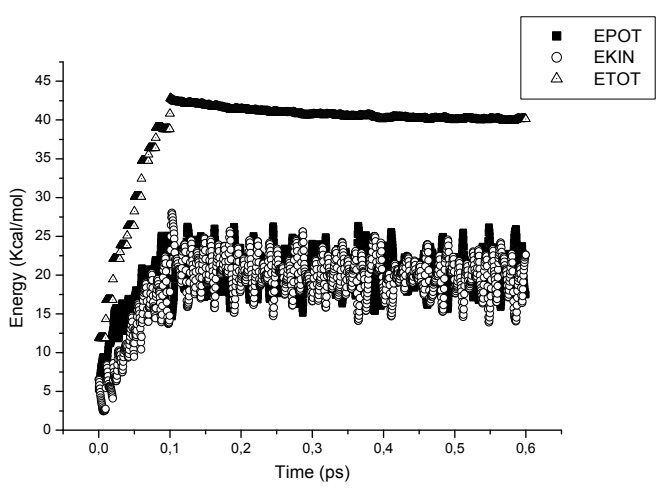

$\left(\mathrm{a}_{1}\right)$

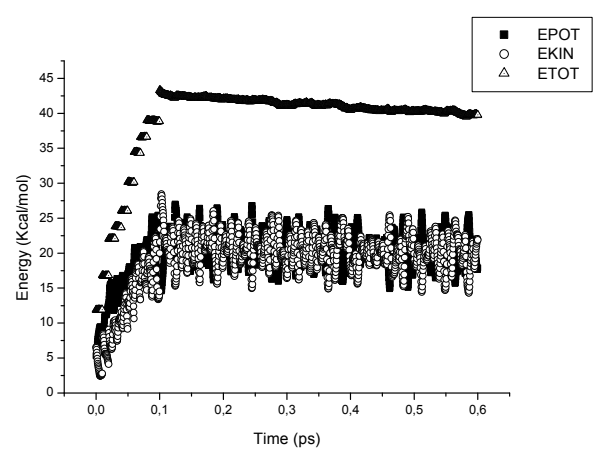

$\left(b_{1}\right)$

Figure 5. Evolution of energies for 5,7-dihydroxy-4-methylcoumarin in TIP3P water molecules with MM+:

$$
\text { (a)MD, (b)LD }
$$

Comparing our results with analysis performed by two semi empirical (Kancheva et al., 2010), we note that the total energies obtained by MD and LD simulations (using AMBER in water) (respectively 41.70 and 41.38 $\mathrm{Kcal} / \mathrm{mol})$ approach that obtained with MNDO $(44.57 \mathrm{Kcal} / \mathrm{mol})$. With $\mathrm{MM}+$ in water, MD and LD total energies $(40.12,39.77 \mathrm{Kcal} / \mathrm{mol})$ are lightly up to the PM3 one $(36.08 \mathrm{Kcal} / \mathrm{mol})$. In gas, the total energies (MD 45.25, LD $45.06 \mathrm{Kcal} / \mathrm{mol}$; under AMBER) are analogous to that with MNDO $(44.57 \mathrm{Kcal} / \mathrm{mol})$. With MM+ in gas, (MD: 42.29 , LD: $40.34 \mathrm{Kcal} / \mathrm{mol}$ ) the situation is similar with those by MM+ in solution. We conclude that our two dynamic methods are suitable for the coumarin system, converge to the MNDO semi-empirical technique and give good results for $\mathrm{MM}+$ force field.

After analyze the evolution of values of dihedrals angles $\mathrm{C}_{10}-\mathrm{O}_{1}-\mathrm{C}_{2}-\mathrm{O}_{11}$ (coco) for coumarin structure, we have observed that the angle is instable in MD simulation, while it has three distinct states of stability in LD calculation with same time intervals (equal to $0.25 \mathrm{ps)}$ in gas. During this simulation run, the torsion angle changes and becomes an anhedral one. The deviations have two states of stability: the first until 0.05 ps for MD and 0.08 ps for LD. The second state has about fully duration of the simulation run in MD and LD calculations (Figure 6).

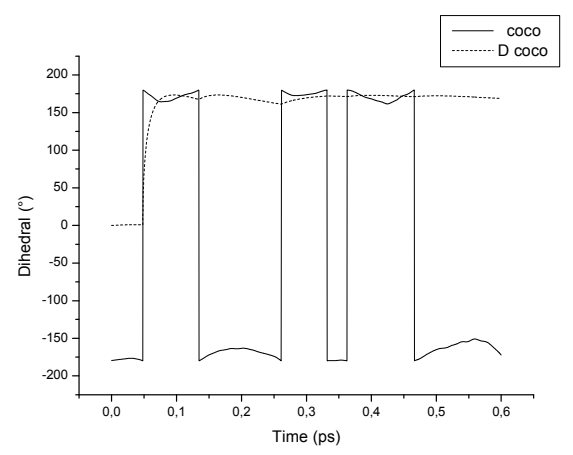

$\left(a_{1}\right)$

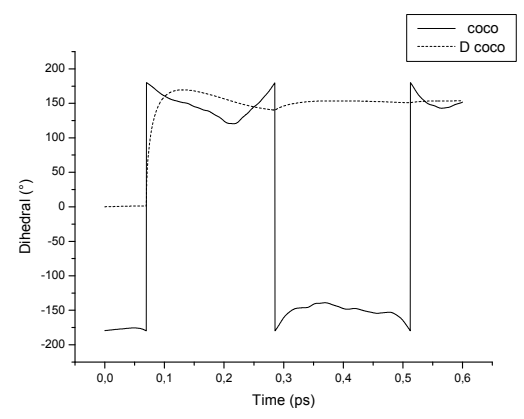

$\left(b_{1}\right)$

Figure 6. Evolution of dihedral angle for 5,7-dihydroxy-4-methylcoumarin in vacuum with AMBER: (a)MD, (b)LD 
The dihedrals are different for the two simulations in solution. In MD calculation, the angle is most anhedral for the period inferior to $0.25 \mathrm{ps}$, and then become more dihedral. In LD simulation, the structure acquires inverse situation. The coumarin undergoes deformations because of the cycle torsion (Figure7). So, we think that the molecule carries an intermediary configuration structure imposed by the delocalization of $\mathrm{C}_{4}=\mathrm{C}_{3}-\mathrm{C}_{2}=\mathrm{O}_{11}$.

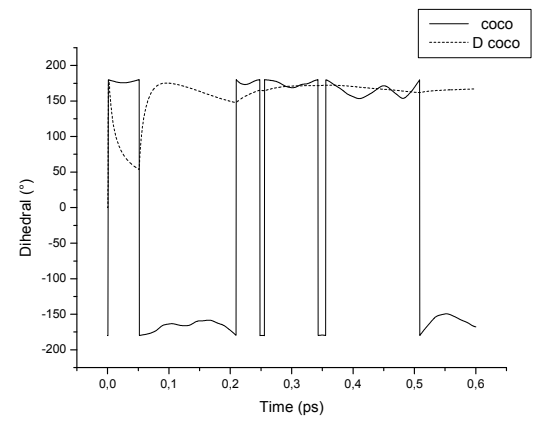

$\left(a_{1}\right)$

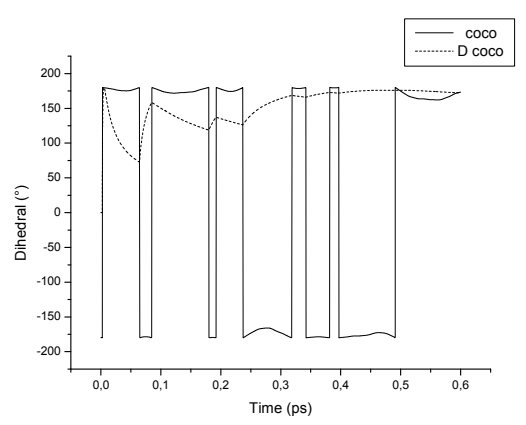

$\left(b_{1}\right)$

Figure 7. Evolution of dihedral angle for 5,7-dihydroxy-4-methylcoumarin in TIP3P water molecules with AMBER: (a)MD, (b)LD

In vacuum (under $\mathrm{MM}+$ ), the dihedrals angles present instability, and oscillate between multiple states (Figure 8).

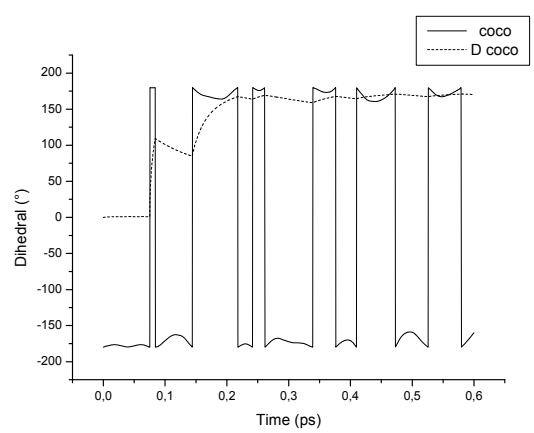

$\left(\mathrm{a}_{2}\right)$

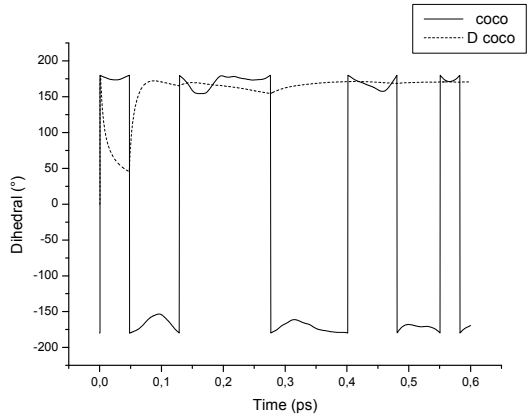

$\left(b_{2}\right)$

Figure 8. Evolution of dihedral for 5,7-dihydroxy-4-methylcoumarin in vacuum with MM+: (a)MD, b)LD

By analogy to calculations with MM+ field in vacuum, in solution the evolution of dihedrals is similar for the two simulations (Figure 9).

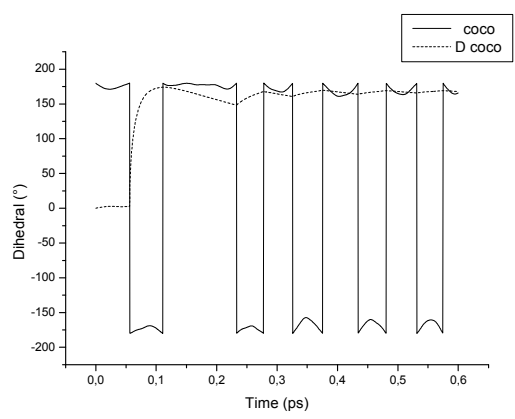

$\left(\mathrm{a}_{2}\right)$

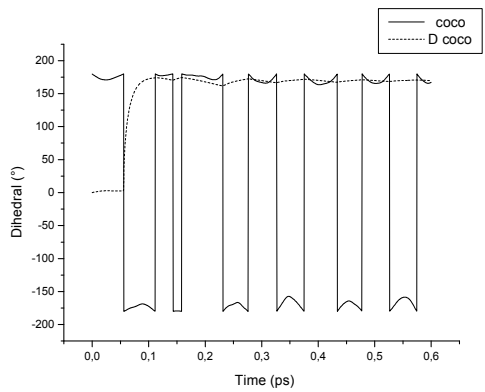

$\left(\mathrm{b}_{2}\right)$

Figure 9. Evolution of dihedral for 5,7-dihydroxy-4-methylcoumarin in TIP3P water molecules with MM+:

(a)MD, (b)LD 
The similarities will appear in both methods (MD, LD) and under the two fields (AMBER and MM+) where the angle changes in these simulations due to the interaction of water molecules. In the actual conditions, the torsion angle turns quickly into anhedral angle for short periods (in six states). Consequently, deviations have an important state of stability while the angle has been affected by the collision. The effect is much represented under MM+ field. So, the structure has been disturbed while the calculation runs. At this transition period, the conformation geometry is conserved.

In the present simulation experiments, the temperature was kept at $300 \mathrm{~K}$. In vacuum, the fluctuations are important and temperature is more stable in MD simulations than in LD ones and is situated near 300K (Figure 10a). In water, the situation is much better. We observe that the resulting trajectories present similarity with fewer fluctuations and the value is flat to $300 \mathrm{~K}$ (Figure 10b).

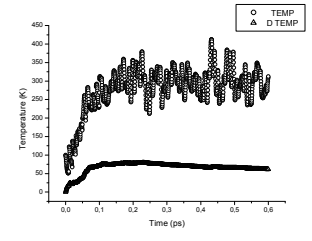

$\left(a_{1}\right)$

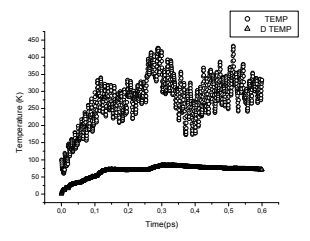

$\left(\mathrm{b}_{1}\right)$

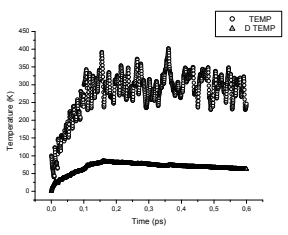

$\left(a_{2}\right)$

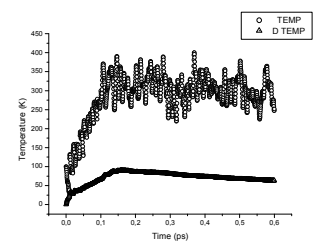

$\left(b_{2}\right)$

Figure 10a. Evolution of temperature for 5,7-dihydroxy-4-methylcoumarin in vacuum : with AMBER (a $\left.a_{1}\right)$ MD, $\left(b_{1}\right) L D ;$ with MM+ $\left(a_{2}\right) M D,\left(b_{2}\right) L D$

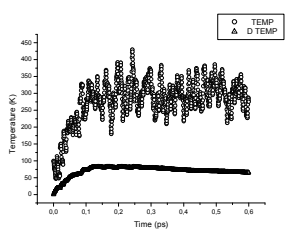

$\left(\mathrm{a}_{1}\right)$

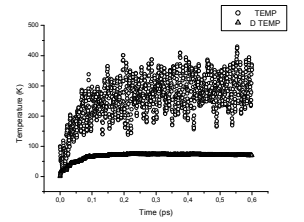

$\left(b_{1}\right)$

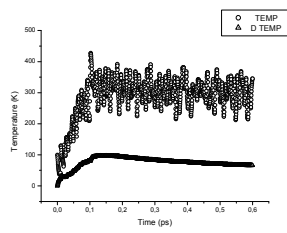

$\left(\mathrm{a}_{2}\right)$

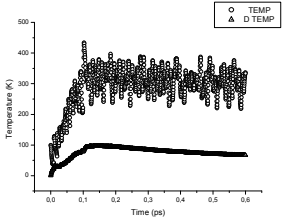

$\left(b_{2}\right)$

Figure 10b. Evolution of temperature for 5,7-dihydroxy-4-methylcoumarin in water : with AMBER (a $\left.)_{1}\right)$ MD, $\left(b_{1}\right) \mathrm{LD}$; with MM+ $\left(\mathrm{a}_{2}\right) \mathrm{MD},\left(\mathrm{b}_{2}\right) \mathrm{LD}$

Furthermore investigations lead us to discuss the energy deviations. In the gas, the RMS Deviations of energies fluctuate around $0.15 \mathrm{ps}$ with average value $(5 \mathrm{Kcal} / \mathrm{mol}$ ) for DEKIN and DEPOT and 9.5 Kcal/mol for DETOT using the AMBER field for the MD method. In LD calculation, the fluctuations were happened around $0.2 \mathrm{ps}$ with resulting values of DETOT: $10.5 \mathrm{Kcal} / \mathrm{mol}$, DEPOT: $6.5 \mathrm{Kcal} / \mathrm{mol}$ and DEKIN: $5.5 \mathrm{Kcal} / \mathrm{mol}$ (Figure $11 \mathrm{a})$. Faraway the maximum, the two curves (DEKIN and DEPOT) converge to same value (5Kcal/mol) in LD simulation.

The same observations are made for DEKIN (MD: 5; LD: $6.5 \mathrm{Kcal} / \mathrm{mol}$ ) under the MM+ field. Under the MM+ field, we observe that DEPOT deviation curve in MD and LD processes show the same value $(5 \mathrm{Kcal} / \mathrm{mol})$. DETOT deviations illustrate similar values $(10 \mathrm{Kcal} / \mathrm{mol})$ for both methods.

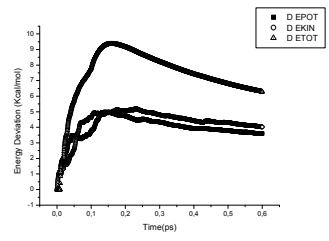

$\left(a_{1}\right)$

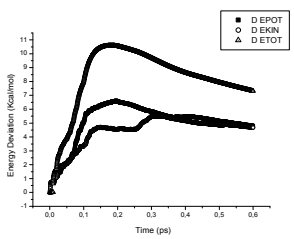

$\left(b_{1}\right)$

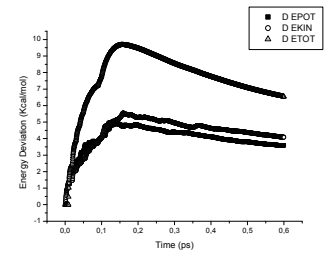

$\left(\mathrm{a}_{2}\right)$

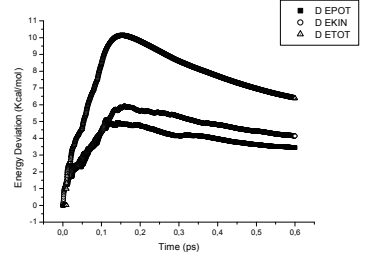

$\left(b_{2}\right)$

Figure 11a. Evolution of energy deviations for 5,7-dihydroxy-4-methylcoumarin in vacuum : with AMBER

$$
\left(a_{1}\right) M D,\left(b_{1}\right) L D ; \text { with MM+ }\left(a_{2}\right) M D,\left(b_{2}\right) L D
$$


In solution, the RMS Deviations of energies fluctuate around $0.15 \mathrm{ps}$ in MD with average values of: DETOT (11 $\mathrm{Kcal} / \mathrm{mol})$, DEKIN and DEPOT $(5.5 \mathrm{Kcal} / \mathrm{mol})$; In LD, they fluctuate around $0.1 \mathrm{ps}$ with average values of DETOT $(8.5 \mathrm{Kcal} / \mathrm{mol})$, DEKIN and DEPOT $(5 \mathrm{Kcal} / \mathrm{mol})$ when using the AMBER field (Figure $11 \mathrm{~b})$. As consequence, the two trajectories (DEKIN and DEPOT) converge to the same value $4 \mathrm{Kcal} / \mathrm{mol}$.

The situation will be unchanged when we used the MM+ field: DETOT (10.5 Kcal/mol), DEKIN (6.5 Kcal/mol) and DEPOT (4.5 Kcal $/ \mathrm{mol})$.

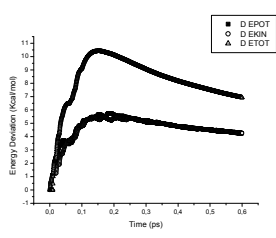

$\left(a_{1}\right)$

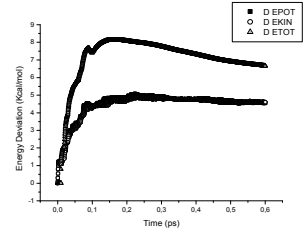

$\left(\mathrm{b}_{1}\right)$
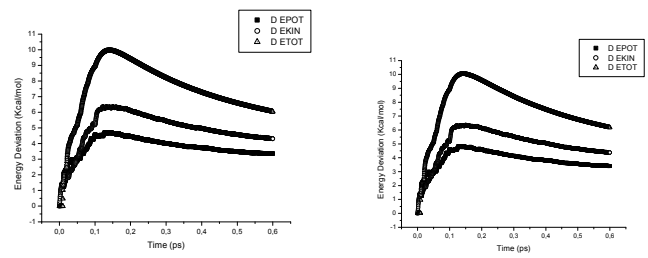

$\left(\mathrm{a}_{2}\right)$

$\left(b_{2}\right)$

Figure 11b. Evolution of energy deviations for 5,7-dihydroxy-4-methylcoumarin in water: with AMBER (a $\left.)_{1}\right)$ MD, $\left(b_{1}\right) L D ;$ with MM+ $\left(a_{2}\right)$ MD, $\left(b_{2}\right)$ LD

DEKIN and DEPOT will stay steady around constant value for both simulations under the two fields (AMBER and $\mathrm{MM}+$ ) in all environments. In solution and under $\mathrm{MM}+$, after the equilibration time (0.5 ps) the corresponding deviations become collinear. In solution, DETOT reach its greatest value $11 \mathrm{Kcal} / \mathrm{mol}$ for both fields. As consequence, the coumarin conformation is more stable in solution.

\subsection{Conformation of Coumarin}

To provide a better estimate of conformations, we should search the conformational space in reasonable computing time. So, we run the simulations at the constant temperature, and then we run a geometry optimization on each structure. In Table 4, we have grouping the following characteristics which are determined after geometry optimization (and dynamic simulations): the dipole moment $(\mu)$, the electrostatic potential (Ep) and the total energy (Et).

Table 4. Properties of 5,7-dihydroxy-4-methylcoumarin at $300 \mathrm{~K}$ with MM+ and AMBER after LD and MD simulations and geometry optimization in two environments: Dipole moment, Electrostatic potential and Total Energy

\begin{tabular}{|c|c|c|c|c|c|}
\hline \multirow{2}{*}{ State } & \multirow{2}{*}{ Method } & \multirow{2}{*}{ Force Field } & \multicolumn{3}{|c|}{ Properties } \\
\hline & & & $\mu(\mathrm{D})$ & Electrostatic potential $(\mathrm{Kcal} / \mathrm{mol})$ & Total Energy $(\mathrm{Kcal} / \mathrm{mol})$ \\
\hline \multirow{4}{*}{ Isolated } & MD & \multirow{2}{*}{ AMBER } & 0.000 & 0.000 & 9.306 \\
\hline & LD & & 0.000 & 0.000 & 9.300 \\
\hline & MD & \multirow{2}{*}{$\mathrm{MM}^{+}$} & 0.908 & 0.340 & 5.316 \\
\hline & LD & & 0.909 & 0.340 & 5.315 \\
\hline \multirow{4}{*}{ Solvated } & MD & \multirow{2}{*}{ AMBER } & 0.000 & 0.000 & 9.306 \\
\hline & LD & & 0.000 & 0.000 & 9.306 \\
\hline & MD & \multirow{2}{*}{$\mathrm{MM}+$} & 0.903 & 0.339 & 5.317 \\
\hline & LD & & 0.909 & 0.339 & 5.317 \\
\hline
\end{tabular}

From these results, we observe that the coumarin has the zero value for the dipole moment and the electrostatic potential when using AMBER field. However under MM+, the dipole moment and the electrostatic potential are unlike of zero and get the same value in both simulations $(\mu=0.908 \mathrm{D}, \mathrm{Ep}=0.34 \mathrm{Kcal} / \mathrm{mol})$.

So, we have plotted the electrostatic potential in $2 \mathrm{D}$ contours with the value obtained after geometry optimization $(0.34 \mathrm{Kcal} / \mathrm{mol})$. Finally, we have compared representation structures to determine low-energy conformations (Figures 12a, 12b). 
The electrostatic potential representation clearly shows that the $\sigma$-withdrawing properties of the oxygen in the lactone reduce the $\pi$-donating and deactivate the ring. So, the molecule grows to be polarizable.

Combining these properties (Table 4) and the representations (Figures 12), we note that under MM+ the coumarin acquires its low-energy value $(5.316 \mathrm{Kcal} / \mathrm{mol})$. At the same time, the molecule has the dipolar characteristic $(\mu=0.908 \mathrm{D})$. On this situation, the molecule reaches its high stable conformation state.

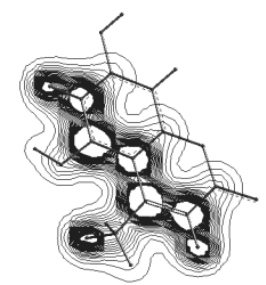

$\left(a_{1}\right)$

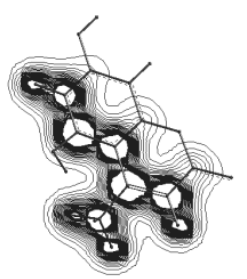

$\left(b_{1}\right)$

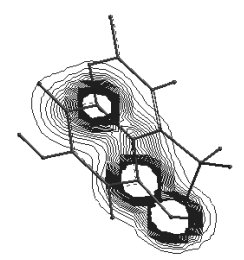

$\left(\mathrm{a}_{2}\right)$

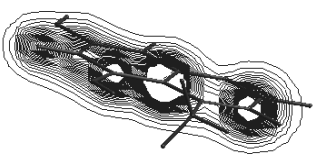

$\left(b_{2}\right)$

Figure 12a. Electrostatic potential 2D representation of Coumarin structure after Dynamic Simulations and geometry optimization in vacuum: with AMBER $\left(a_{1}\right) M D,\left(b_{1}\right) L D$; with MM+ $\left(a_{2}\right) M D,\left(b_{2}\right) L D$

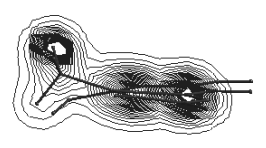

$\left(\mathrm{a}_{1}\right)$

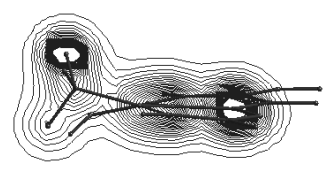

$\left(b_{1}\right)$

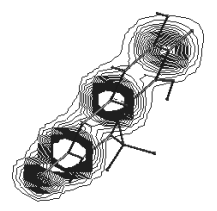

$\left(\mathrm{a}_{2}\right)$

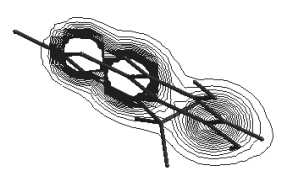

$\left(b_{2}\right)$

Figure 12b. Electrostatic potential 2D representation of Coumarin structure after Dynamic Simulations and geometry optimization in water: with AMBER $\left(a_{1}\right) M D,\left(b_{1}\right) L D$; with MM+ $\left(a_{2}\right) M D$, $\left(b_{2}\right) L D$

\section{Conclusion}

We have studied the evolution of the geometry optimized properties for 5,7-dihydroxy-4-methylcoumarin. We have chosen the MD and LD methods to predict much better the characteristics. The geometries, interaction energies, bonds, angles, stretch-bends, electrostatic and the VDW interactions were carried out in solution and in gas phase. We have calculated the thermodynamic and structural properties for both the two force fields Amber and $\mathrm{MM}+$.

The total energy is more stable in MD simulations under the AMBER and MM+ fields. Under AMBER and $\mathrm{MM}+$ in water, we note that the total energies obtained by MD and LD simulations are similar. In gas, the situation is similar. We conclude that our two dynamic methods are suitable for the coumarin system, and give good results for $\mathrm{MM}+$ force field.

The deviations of energies stay steady around constant value for both simulations under the two fields (AMBER and $\mathrm{MM}+$ ) in all environments. In solution, the total energy deviation reaches its greatest value for both fields. As consequence, the coumarin conformation is more stable in solution.

The torsion angle changes in these simulations due to the interaction of water molecules and turns quickly into anhedral angle for short periods. At this transition period, the conformation geometry is conserved. LD method is more effective for calculate the dihedral angle with $\mathrm{MM}+$ field in solution environment.

We conclude that our simulation under MM+ field gives best results; the coumarin acquires the low-energy and has the dipolar characteristic. So, the molecule reaches its high stable conformation state in solution environment under MM+ field.

\section{References}

Borges, F., Roleira, F., Milhazes, N., Santana, L., \& Uriarte, E. (2005). Simple Coumarins Andanalogues in Medicinal Chemistry: Occurrence, Synthesis and Biological Activity. Curr. Med. Chem., 12(8), 887-916. http://dx.doi.org/10.2174/0929867053507315 
Cornell, W. D., Cieplak, P., Bayly, C. I., Gould, I. R., Merz, K. M. Jr., Ferguson, D. M., ... Kollman, P. A. (1995). A Second Generation Force Field for the Simulation of Proteins, Nucleic Acids, and Organic Molecules. J. Am. Chem. Soc., 117, 5179-5197. http://dx.doi.org/10.1021/ja00124a002

Goel, A., Prasad, A. K., Parmar, V. S., Ghosh, B. S., \& Saini, N. (2007). 7,8-Dihydroxy-4-methylcoumarin Induces Apoptosis of Human Lung Adenocarcinoma Cells by ROS-Independent Mitochondrial Pathway Through Partial Inhibition of ERK/MAPK Signaling. FEBS Lett., 581, 2447-2454. http://dx.doi.org/10.1016/j.febslet.2007.04.052

Hoult, J. R. S., \& Payá, M. (1996). Pharmacological and Biochemical Actions of Simple Coumarins: Natural Products with Therapeutic Potential. Gen. Pharmacol., 27(4), 713-722. http://dx.doi.org/10.1016/0306-3623(95)02112-4

Kancheva, V. D. (2009). Phenolic Antioxidants: Antiradical and Antioxidant Activity: Comparable Study. Eur. J. Lipid. Sci. Technol., 111, 1072-1089. http://dx.doi.org/10.1002/ejlt.200900005

Kancheva, V. D., Saso, L., Boranova, P. V., Khan, A., Saroj, M. K., Pandey, M. K., ... Parmar, V. S. (2010). Structure-Activity Relationship of Dihydroxy-4-Methylcoumarins as Powerful Antioxidants: Correlation between Experimental and Theoretical Data and Synergistic Effect. Biochimie., 92(9), 1089-1100. http://dx.doi.org/10.1016/j.biochi.2010.06.012

Karplus, M., \& Petsko, G. A. (1990). Molecular Dynamics Simulations in Biology. Nature., 347, 631-639. http://dx.doi.org/10.1038/347631a0

Kumar, S., Singh, B. K., Tyagi, R., Jain, S. K., Sharma, S. K., Prasad, A. K., ... Parmar, V. S. (2005). Mechanism of Biochemical Action of Substituted 4-Methylcoumarins. Part 11: Comparison of the Specificities of Acetoxy Derivatives of 4-Methylcoumarin and 4-Phenylcoumarin to Acetoxycoumarins: Protein Transacetylase. Bioorg. Med. Chem., 13, 4300-4305. http://dx.doi.org/10.1016/j.bmc.2005.04.023

MacKerrell, A. D. Jr., Bashford, D., Bellott, R. L., Dunbrack, R. L. Jr., Evanseck, J. D., Field, M. J., ... D., Karplus, M. (1998). All-Atom Empirical Potential for Molecular Modeling and Dynamics Studies of Proteins. J. Phys. Chem B., 102, 3586-3616. http://dx.doi.org/10.1021/jp973084f

Mesli, F., \& Mahboub, R. (2010). Evolution of Thermodynamic and Structural Properties of Coumarins by Molecular Dynamics Simulation. Res. J. Pharm. Biol. Chem. Sci., 1, 83-92.

Pedersen, J. Z., Oliveira, C., Incerpi, S., Kumar, V., Fiore, A. M., De Vito, P., ... Saso, L. (2007) Antioxidant $\begin{array}{lllll}\text { Activity of 4-Methylcoumarins. J. Pharm. Pharmacol., 59, 1721-1728. } & \text {. }\end{array}$ http://dx.doi.org/10.1211/jpp.59.12.0015

Ponder, J. W., \& Case, D. A. (2003). Force Fields for Protein Simulations. Adv. Protein. Chem., 66, 27-85. http://dx.doi.org/10.1016/S0065-3233(03)66002-X

Sabbaghzadeh, R., Monajjemi, M., Mollaamin, F., \& Oryan, S. (2010). The Effect of Monte Carlo, Molecular Dynamic and Langevin Dynamic. Simulation and Computational Calculations on Insulin-Like Growth Factor-1 (IGF-1). J. Cell. Mol. Res., 2(2), 93-102.

Shan, Z. X., \& Xiao, C. G. (2004). Synthesis of Hymecromone Derivatives Containing Chiral 1,1'-Bi-2-Naphthyl Moiety for Dual-Mode Molecular Switch. Chin. Chem. Lett., 15(8), 892-894.

van Gunsteren, W. F., \& Berendsen, H. J. C. (1990). Computer Simulation of Molecular Dynamics: Methodology, Applications and Perspectives in Chemistry. Angew. Chem. Int. Ed. Engl., 29(9), 992-1023. http://dx.doi.org/10.1002/anie.199009921

Weiner, S. J., Kollman, P. A., Case, D. A., Singh, U. C., Ghio, C., Alagona, G., ... Weiner, P. (1984). A New Force Field for Molecular Mechanical Simulation of Nucleic Acids and Proteins. J. Am. Chem. Soc., 106, 765-784. http://dx.doi.org/10.1021/ja00315a051

Wheelock, C. E. (1959). The Fluorescence of Some Coumarins. J. Am. Chem. Soc., 81(6), 1348-1352. http://dx.doi.org/10.1021/ja01515a021 